\title{
Galectins, Eosinophiles, and Macrophages May Contribute to Schistosoma japonicum Egg-Induced Immunopathology in a Mouse Model
}

\author{
Zhanhong Ye ${ }^{1}$, Shiguang Huang ${ }^{2 *}$, Yanxia Zhang ${ }^{1}, X u$ Mei $^{3}$, Huanqin Zheng ${ }^{1}$, Meiyu Li ${ }^{4}$, \\ Jianhuang $\mathrm{Chen}^{4}$ and Fangli $\mathrm{Lu}^{1 *}$ \\ 'Department of Parasitology, Zhongshan School of Medicine, Key Laboratory of Tropical Disease Control of Ministry of \\ Education, Sun Yat-sen University, Guangzhou, China, ${ }^{2}$ School of Stomatology, Jinan University, Guangzhou, China, \\ ${ }^{3}$ Artemisinin Research Center and Institute of Science and Technology, Guangzhou University of Chinese Medicine, \\ Guangzhou, China, ${ }^{4}$ Medical Experimental Teaching Center, Zhongshan School of Medicine, Sun Yat-sen University, \\ Guangzhou, China
}

Schistosomiasis is a severe public health problem, which can cause tissue fibrosis and can even be fatal. Previous studies have proven that galectins and different kinds of cells involve in the regulation of tissue fibrosis process. In this study, outbred Kunming mice were infected with Schistosoma japonicum (S. japonicum). Our results showed that compared with uninfected mice, there were severe egg granulomatous inflammation and tissue fibrosis in the livers, spleens, and large intestines of $S$. japonicum-infected mice at 8 weeks post-infection (p.i.), and the number of eosinophils by hematoxylin and eosin staining and CD68 macrophage-positive area by immunohistochemical staining were significantly increased. Detected by using quantitative real-time reverse transcription-polymerase chain reaction (qRT-PCR), at 8 weeks after S. japonicum infection, the mRNA expression levels of galectin (Gal)-1, Gal-3, CD69, eosinophil protein $X(E P X)$, and chitinase 3-like protein 3 (Ym1) were significantly increased in liver, spleen, and large intestine; eotaxin-1 (CCL11) and eosinophil cationic protein were significantly increased in both liver and spleen; eotaxin-2 (CCL24) and Arginase1 (Arg1) were significantly increased in both spleen and large intestine; and CD200R was significantly increased in both liver and large intestine. However, interleukin (IL)-1B and inducible nitric oxide synthase (iNOS) were only significantly increased in liver. The M2/M1 ratio of CD200R/CD86 genes was significantly increased in liver, and ratios of $Y m 1 / I L-1 \beta$ and $Y m 1 / i N O S$ were significantly increased in liver, spleen, and large intestine of S. japonicum-infected mice. Ex vivo study further confirmed that the levels of Gal-1, Gal-3, CD200R, Arg1, and Ym1 were significantly increased, and the ratios of CD200R/CD86 and Ym1/IL-1 $\beta$ were significantly increased in peritoneal macrophages isolated from $S$. japonicum-infected mice at 8 weeks p.i. In addition, correlation analysis showed that significant positive correlations existed between mRNA levels of Gal-1/Gal-3 and EPX in liver, between Gal-3 and Ym1 in both liver and large intestine, and between Gal-3 and CD200R in peritoneal macrophages of S. japonicum-infected mice. Our data suggested that Gal-1, Gal-3, eosinophils, and macrophages are likely involved in the development of egg granulomatous response and fibrosis induced by S. japonicum infection.

Keywords: Schistosoma japonicum, mice, immunopathology, Gal-1, Gal-3, eosinophils, macrophages 


\section{INTRODUCTION}

Schistosomiasis is a zoonotic parasitic disease caused by Schistosoma spp. among human beings and animals, which affects nearly 250 million population worldwide (1). Three main species of Schistosoma-Schistosoma japonicum (S. japonicum), S. mansoni, and $S$. haematobium-have the most wide prevalence globally. S. japonicum, mainly distributed in China, the Philippines, and Indonesia, can cause severe chronic schistosomiasis japonica (2). The eggs of S. japonicum immigrate and deposit in liver and intestine tissues, which recruit macrophages, neutrophils, and eosinophils into the tissues during granulomatous reactions (3), and fibrotic deposits distributed around granulomas results in pipestem fibrosis (4). However, so far, the mechanism of tissue fibrosis caused by $S$. japonicum remains not fully understood.

Eosinophils are end effector cells involved in host protection against helminth infection (5). It has been reported that granuloma eosinophils are highly activated and produce the majority of Th2 cytokines in granulomatous inflammation, which may be important determiners of immunopathology in murine schistosomiasis caused by S. mansoni (6). The eosinophil granulocytes contain four major proteins, i.e., eosinophil cationnic protein [ECP; (7)], eosinophil peroxidase [EPO; (8)], eosinophil protein X (EPX)/eosinophil-derived neurotoxin (9), and major basic protein [MBP; (10)]. Eotaxin is an essential regulator of eosinophil trafficking during healthy conditions (11) and inflammation (12). EPO and MBP are correlated with cytokine responses of macrophages and $\mathrm{CD} 4^{+} \mathrm{T}$ cells, and mice deficient in either EPO or MBP developed significantly higher worm burdens of Litomosoides sigmodontis than wild-type mice (13). In patients infected with S. haematobium, both ECP and EPX protein levels are higher in association with rubbery papules in genital lavage, which may serve as markers for a potential early-stage inflammatory lesion in female genital schistosomiasis (14). In addition, increased levels of ECP, EPO, and EPX in sera of patients are related to cystic fibrosis (15). A study demonstrated that primary murine hepatic myofibroblasts derived from granulomas of $S$. mansoni-infected mice can produce interleukin (IL)-5 and eotaxin, which may contribute to maintenance of local eosinophilia in schistosomal hepatic granulomas (16). Eotaxin/eotaxin-1 (CCL11) drives tissue infiltration of eosinophils and mast cells, which can promote pathogenesis in patients with diopathic retroperitoneal fibrosis (17). Thus, eosinophils play an essential role in granulomas and tissue fibrosis.

Macrophages are divided into classically activated macrophages (M1) and alternatively activated macrophages (M2). M1 type macrophages can release monocyte chemotactic protein- $1 \beta$ and inducible nitric oxide synthase (iNOS) and mainly promote inflammatory reaction, whereas M2 type macrophages play a role in the immunoregulation and tissue remodeling (18). It has been reported that hepatitis B virusmediated liver disease is associated with high level of infiltrated human macrophages with M2-like activation phenotype (19). M2 polarization has been proven to be associated with the process of tissue fibrosis (20). During S. japonicum infection, macrophages and schistosome soluble egg antigen (SEA) interaction play a critical role in regulation of host immune responses (21).

Galectins are a family of carbohydrate-binding proteins that are involved in many physiological functions, and 15 members have been identified in various cells and tissues (22). They involve in immunity (23), apoptosis (24), immune tolerance, inflammation (25), and cell adhesion (26). Galectins play important roles in the development of acute inflammation as well as chronic inflammation (27). Both galectin (Gal)-1 and Gal-3 are distributed widely in different cells and tissue types, including innate and adaptive immune cells $(23,28)$. Gal-1 and Gal-3 facilitate the proliferation of hepatic stellate cells (HSCs) and play an important role in liver fibrosis (29). Some researchers proved that down-regulated expression levels of $\alpha$-smooth muscle actin (SMA) and transforming growth factor (TGF)- $\beta 1$ and improved liver fibrosis have been observed in silencing Gal-1 mouse models (30). Macrophage-derived Gal-3 is fundamental for the activation of myofibroblasts (31). S. mansoni-infected Gal- $3^{-/-}$mice had an increase of monocytes and eosinophils in the granulomas from acute and chronic phases of the disease (32). Granuloma-derived stromal cells from S. mansoni-infected Lgals $3^{-/}$mice express lower levels of $\alpha$-SMA and eotaxin and higher levels of IL-4 and significant inflammatory infiltration than Lgals $3^{+/+}$infected mice (33). Gal-3 inhibitor is proven to significantly decrease the percentage of liver and kidney fibrosis area in a non-alcoholic steatohepatitis mouse model (34). However, the concrete mechanism of how galectins influence schistosomiasis fibrosis remains unknown.

To assess the functions of galectins, eosinophils, and macrophages during the stage of S. japonicum egg depositioninduced inflammation and fibrosis, in the present study, we compare the expression levels of Gal-1, Gal-3, eosinophil chemoattractant (CCL11 and eotaxin-2 [CCL24]), eosinophil marker (CD69), eosinophil granule proteins (ECP and EPX), M1 macrophage markers (CD86, IL-1 $\beta$, and iNOS), and M2 macrophage markers (CD200R, Arginase1 [Arg1], and chitinase 3-like protein 3 [Ym1]) in the livers, spleens, large intestines, and peritoneal macrophages of mice with chronic schistosomiasis japonica. Based on the relationship among galectins, eosinophils, macrophage polarization, and pathology of schistosomiasis japonica, our data demonstrated that Gal-1, Gal-3, eosinophils, and macrophages play important roles in $S$. japonicum egg deposition-induced immune response and fibrosis in advanced schistosomiasis japonica mouse model.

\section{MATERIALS AND METHODS}

\section{Ethics Statement}

In vivo experiments were approved by the Animal Experimentation Ethics Committee of Zhongshan School of Medicine on Laboratory Animal Care at Sun Yat-sen University (No. 2016-081) and were carried out in strict accordance with institutional Guidelines for Care and Use of Laboratory Animals. 


\section{Mice and Parasite Infection}

Female Kunming mice (outbred, 6-8 weeks old) were purchased from the Animal Facility of Sun Yat-sen University. Oncomelania hupensis snails were obtained from the National Institute of Parasitic Diseases, Chinese Center for Disease Control and Prevention (Shanghai, China). Forty-six mice were used in this experiment, which were divided into two groups, i.e., a naive group and an infected group, each containing 23 mice.
Infected mice were infected percutaneously with 30 cercariae of S. japonicum.

\section{Histopathology}

At 8 weeks post-infection (p.i.), naive mice and S. japonicuminfected mice were euthanatized by $\mathrm{CO}_{2}$ asphyxiation and their livers, spleens, and large intestines were harvested. Samples were fixed in 10\% buffered natural formaldehyde

TABLE 1 | Primer sequences of genes used for quantitative real-time reverse transcription-polymerase chain reaction assays.

\begin{tabular}{|c|c|c|c|}
\hline Genes & Forward primer $\left(5^{\prime} \rightarrow 3^{\prime}\right)$ & Reverse primer $\left(5^{\prime} \rightarrow 3^{\prime}\right)$ & Accession \\
\hline GAPDH & АCTCCACTCACGGCAAATTC & TCTCCATGGTGGTGAAGACA & NM_001289726.1 \\
\hline $\mathrm{IL}-1 \beta$ & AATGACCTGTTCTITGAAGTTGA & TGATGTGCTGCTGCGAGATITGAAG & NM_008361.4 \\
\hline iNOS & GTTCTCAGCCCAACAATACAAGA & GTGGACGGGTCGATGTCAC & NM_010927.4 \\
\hline Ym1 & AGAAGGGAGTTCAAACCTGGT & GTCTTGCTCATGTGTGTAAGTGA & NM_009892.3 \\
\hline Arg1 & CAGAAGAATGGAAGAGTCAG & CAGATATGCAGGGAGTCACC & NM_007482.3 \\
\hline Gal-1 & CGCCAGCAACCTGAATC & GTCCCATCTTCCTTGGTGTTA & NM_008495.2 \\
\hline Gal-3 & AACACGAAGCAGGACAATAACTGG & GCAGTAGGTGAGCATCGTTGAC & NM_010705.3 \\
\hline ECP & ATCCAAGTGGCTTGTGCAGTGAC & TAAGGTGTTCTCCTCCGACTGGTG & XM_021155370.1 \\
\hline EPX & CACGAACCAGCGATTCCAGGAC & GCTCAGCGGCTAGGCGATTG & NM_007946.2 \\
\hline CCL11 & CAGATGCACCCTGAAAGCCA & CAGGTGCTITGTGGCATCCT & NM_011330.3 \\
\hline CCL24 & CTCAGATGGTGGTGTGCTTGCC & TCCTCTTGССTCTGCCTTCTGG & NM_019577.5 \\
\hline CD69 & GGGCTGTGTTAATAGTGGTCCTC & CTTGCAGGTAGCAACATGGTGG & NM_001033122 \\
\hline CD86 & ACGTATTGGAAGGAGATTACAGCT & TCTGTCAGCGTTACTATCCCGC & NM_019388 \\
\hline CD200R & TGTGAGACAGTAACACCTGAAGG & TGCCATTGCCTCACAGACTGCA & NM_021325 \\
\hline
\end{tabular}

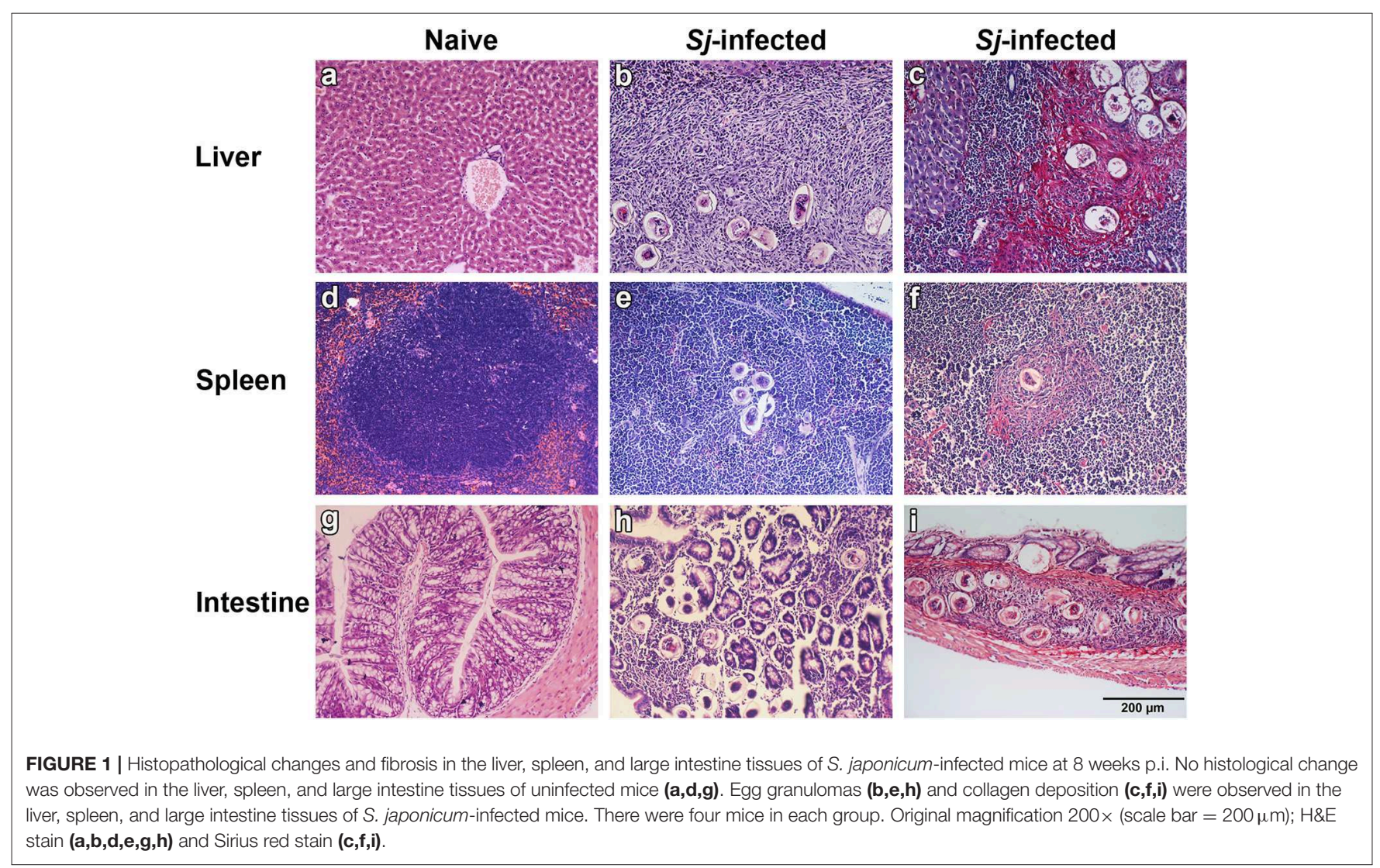


(Guangzhou Chemical Reagent Factory, China) for over $48 \mathrm{~h}$. The paraffin-embedded tissues from each mouse were sectioned at $4 \mu \mathrm{m}$ and prepared for hematoxylin and eosin (H\&E) staining (Sigma-Aldrich, Shanghai, China). The histopathological changes of liver, spleen, and large intestine from each mouse were determined under $200 \times, 400 \times$, or $1000 \times$ magnification in three noncontiguous sections. The number of eosinophils were quantified using images captured with a digital camera system under $1000 \times$ magnification and analyzed by using Image-Pro Plus (Image Z1 software, version 6.0, Media Cybernetics, MD, United States), and the density of eosinophils was expressed as the number of eosinophils per square millimeter.

\section{Sirius Red Staining}

To detect the deposition of collagen fiber from different tissues, paraffin-embedded liver, spleen, and large intestine from each mouse were sectioned at $4 \mu \mathrm{m}$ and stained by Sirius red stain kit (Beijing Leagene Biotchnology Co., Ltd., China).

\section{Immunohistochemical Staining}

The paraffin-embedded liver, spleen, and large intestine sections $(4-\mu \mathrm{m})$ were deparaffinized and rehydrated in distilled water. Heat-induced antigen retrieval was carried out in an $800-\mathrm{W}$ microwave oven for $30 \mathrm{~min}$. Sections were treated with $3 \%$ hydrogen peroxide in methanol for $10 \mathrm{~min}$ at $37^{\circ} \mathrm{C}$ and then incubated in $10 \%$ normal goat serum with $1 \%$ bovine serum albumin (Sigma-Aldrich) in PBS ( $\mathrm{pH} \mathrm{7.4)} \mathrm{for} 10 \mathrm{~min}$ at room temperature to block non-specific binding. After washing with PBS, sections were incubated with rabbit anti-Gal-1 (1:500 dilution; Wuhan Boster Biological Engineering Co., Ltd., Wuhan, China), rabbit anti-Gal-3 polyclonal antibody (IgG1; 1:200 dilution; Bioss, Beijing, China), or rabbit antiCD68 (1:200 dilution; Wuhan Boster Biological Engineering Co., Ltd.) overnight at $4^{\circ} \mathrm{C}$. Those sections incubated with secondary antibodies alone were used as isotype controls. Immunohistochemical staining was then performed with a streptavidin-biotin-peroxidase complex kit and developed with diaminobenzidine tetrahydrochloride (Beijing Zhongshan Golden Bridge Biotechnology, Beijing, China). The sections were counterstained with hematoxylin and positive cells were identified by dark-brown staining under light microscopy. The immunohistochemistry signal (positive areas) of CD68 were quantified using images captured with a digital camera system under $400 \times$ magnification and analyzed by using Image-Pro Plus (Image Z1 software, version 6.0, Media Cybernetics, MD, United States).

\section{Isolation of Murine Peritoneal Macrophages}

At 8 weeks p.i., eight naive mice and eight $S$. japonicum-infected mice were injected intraperitoneally with $2 \mathrm{ml} 3 \%$ thioglycollate broth (Sigma-Aldrich) solution in PBS once daily for 3 days, and animals were sacrificed and their peritoneal lavage fluid were spun at $800 \mathrm{~g}$ at $4^{\circ} \mathrm{C}$ for $5 \mathrm{~min}$, and the pelleted peritoneal macrophages were resuspended and seeded at $5 \times 10^{5}$ cells/well in 12-well plates (Corning, NY, United States). After $4 \mathrm{~h}$ at $37^{\circ} \mathrm{C}$ in a $5 \% \mathrm{CO}_{2}$ atmosphere, cells were washed and collected. Samples were stored at $-80^{\circ} \mathrm{C}$ until subjected to further analysis.

\section{RNA Isolation, cDNA Synthesis, and Quantitative Real-Time Reverse Transcription-Polymerase Chain Reaction (qRT-PCR)}

Total RNA was extracted from about $100 \mathrm{mg}$ of mouse liver, spleen, and large intestine tissues of each mouse or peritoneal macrophages isolated from each mouse using an RNA Extraction Kit (Takara Bio Inc., Shiga, Japan). The quality and quantity of RNA were determined by NanoDrop 2000 spectrophotometer (Thermo Fisher, Waltham, MA, United States). First-strand cDNA was constructed from $1.0 \mu \mathrm{g}$ of total RNA with oligo(dT)

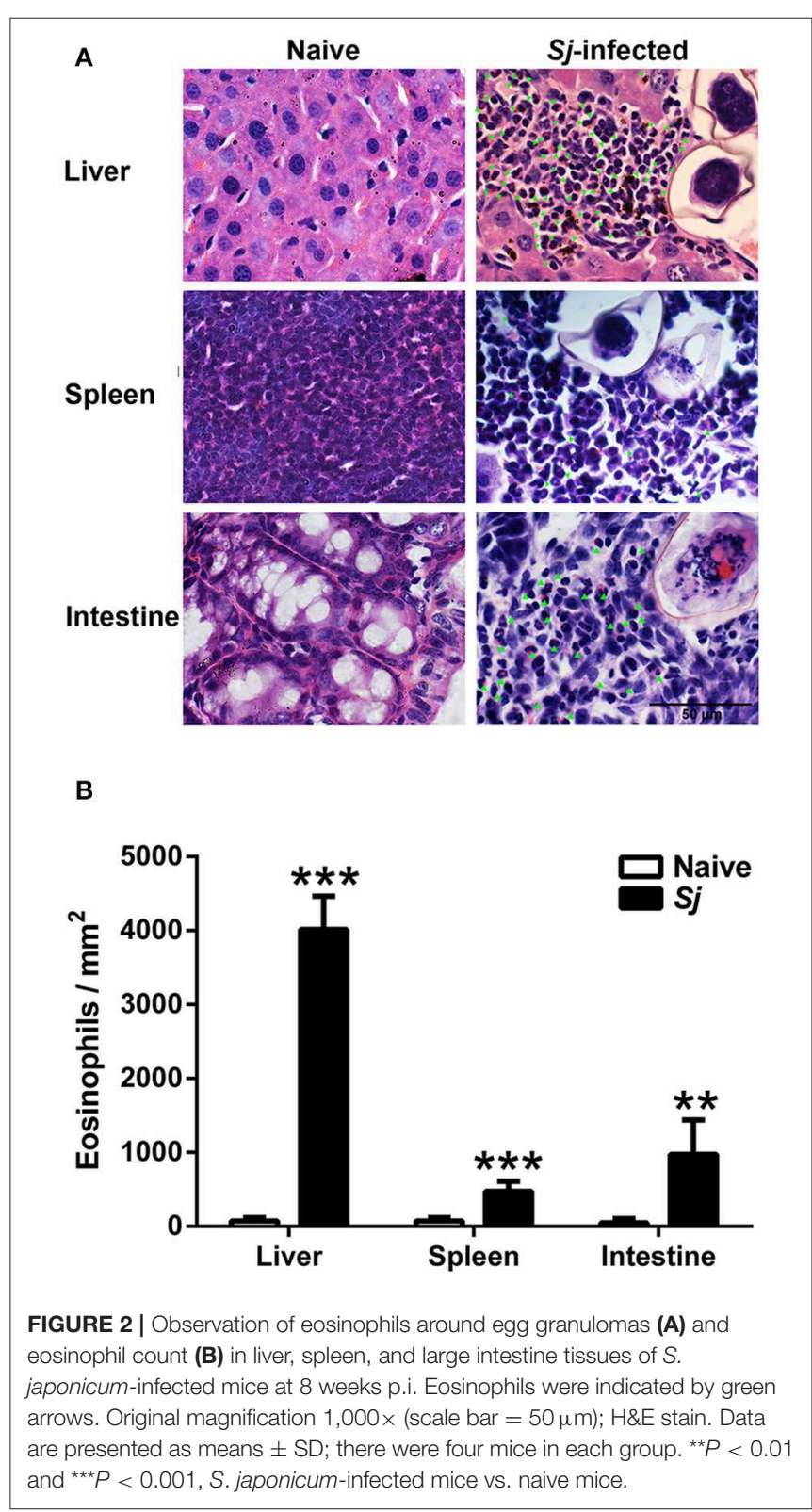


as primers using a PrimeScript 1st Strand cDNA Synthesis Kit (Takara Bio Inc.). The primer sequences are listed in Table 1. To determine mRNA levels of CD69, CCL11, CCL24, ECP, and EPX in liver, spleen, and large intestine tissues and mRNA levels of CD86, CD200R, Gal-1, Gal-3, Arg1, Ym1, IL-1 $\beta$, and iNOS in liver, spleen, and large intestine tissues, and peritoneal macrophages, qRT-PCR measurements were performed using SYBR Green QPCR Master Mix (Takara Bio Inc.). Briefly, a total of $10 \mu \mathrm{l}$ reaction mixture contained $5.0 \mu \mathrm{l}$ of SYBR $\mathbb{R}$ Premix Ex TaqTM $(2 \times), 0.5 \mu \mathrm{l}$ of each primer $(10 \mathrm{pM}), 3.0 \mu \mathrm{l}$ of $\mathrm{dH}_{2} \mathrm{O}$, and $1.0 \mu \mathrm{l}$ of cDNA $(0.2 \mu \mathrm{g} / \mu \mathrm{l})$. Amplification was pre-denaturized for $30 \mathrm{~s}$ at $95^{\circ} \mathrm{C}$, followed by 43 cycles of $5 \mathrm{~s}$ at $95^{\circ} \mathrm{C}$ and $20 \mathrm{~s}$ at $60^{\circ} \mathrm{C}$ with a CFX96 real-time PCR detection system (Bio-Rad Laboratories, Hercules, CA, United States). The mRNA expression levels of CD69, CD86, CD200R, galectins, cytokines, and chemokines were normalized to that of mouse housekeeping gene, GAPDH. The results were expressed as fold change compared with uninfected mice.

\section{Statistical Analysis}

Statistical analysis was performed using SPSS Statistics version 22.0 (SPSS Inc., Chicago, IL, United States). Data are presented as means \pm standard deviation (SD) with at least three independent biological replicates. Student $t$-test was used to compare data between different groups. Pearson's correlation coefficient was used to analyze correlations between the levels of CD69, CD86, CD200R, galectins, cytokines, and chemokines. $P<0.05$ was considered statistically significant.

\section{RESULTS}

\section{The Pathology and Fibrosis in the Livers, Spleens, and Large Intestines of Mice Infected With S. japonicum}

The livers, spleens, and large intestines of both naive and S. japonicum-infected mice were examined histologically at 8 weeks p.i. The results showed that the sections of livers, spleens, and large intestines from uninfected control mice were negative for pathological changes and egg granulomas. However, the sections from infected mice showed severe histological change and S. japonicum egg granulomas with eosinophils and other inflammation cells gathering in the tissues of liver, spleen, and large intestine. Classical pigmented Kupffer cells were

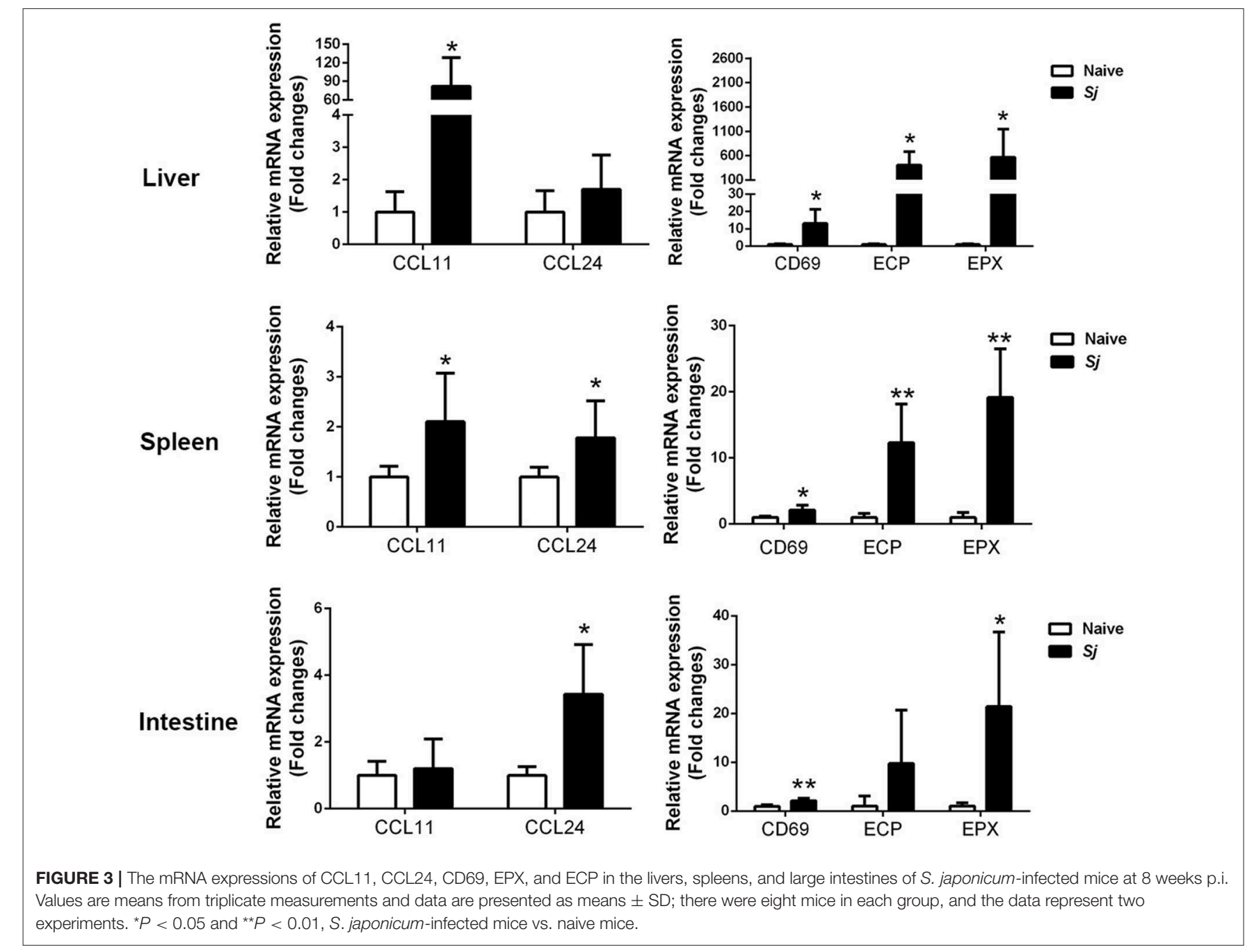


found around liver granulomas (Figures 1a,b,d,e,g,h). Sirius red staining showed that large amount of collagen deposition was observed around granulomas in liver, spleen, and large intestine tissues of $S$. japonicum-infected mice (Figures 1c,f,i).

\section{Eosinophils in the Livers, Spleens, and Large Intestines of S. japonicum-Infected Mice}

By H\&E staining, compared with uninfected controls, there was significantly increased eosinophil infiltrate around egg granulomas at 8 weeks p.i. (Figure 2A). Quantitative analysis showed that the number of eosinophils were significantly increased in the livers $(P<0.001)$, spleens $(P<0.001)$, and large intestines $(P<0.01)$ of $S$. japonicum-infected mice compared with uninfected controls (Figure 2B).

\section{The mRNA Levels of CD69, CCL11, CCL24, ECP, and EPX in the Livers, Spleens, and Large Intestines of S. japonicum-Infected Mice}

Compared with uninfected controls, S. japonicum-infected mice presented significantly elevated mRNA expression levels of
CCL11, ECP, EPX, and CD69 in the livers $(P<0.05)$; significantly elevated levels of CCL11 $(P<0.05)$, CCL24 $(P<0.05)$, CD69 $(P<0.05)$, EPX $(P<0.01)$, and ECP $(P<0.01)$ in the spleens; and significantly elevated levels of CCL24 $(P<0.05)$, EPX $(P$ $<0.05)$, and CD69 $(P<0.01)$ in the large intestines at 8 weeks p.i. (Figure 3).

\section{Immunohistochemical Staining and mRNA Expression of Gal-1 and Gal-3 in the Livers, Spleens, and Large Intestines of \\ S. japonicum-Infected Mice}

There were only a few Gal-1- and Gal-3-positive cells observed in the liver, spleen, and large intestine of uninfected mice. However, a large amount of Gal-1 and Gal-3 positive cells labeled dark brown, especially around the granulomas, were presented in the liver, spleen, and large intestine of S. japonicum-infected mice (Figures 4A,B). The Gal-1 and Gal-3 mRNA expression levels in the liver, spleen, and large intestine tissues were measured. Compared with uninfected controls, $S$. japonicum-infected mice presented significantly elevated Gal-1 and Gal-3 levels in the livers $(P<0.05$ and $P<0.01$, respectively), spleens $(P<0.01)$,

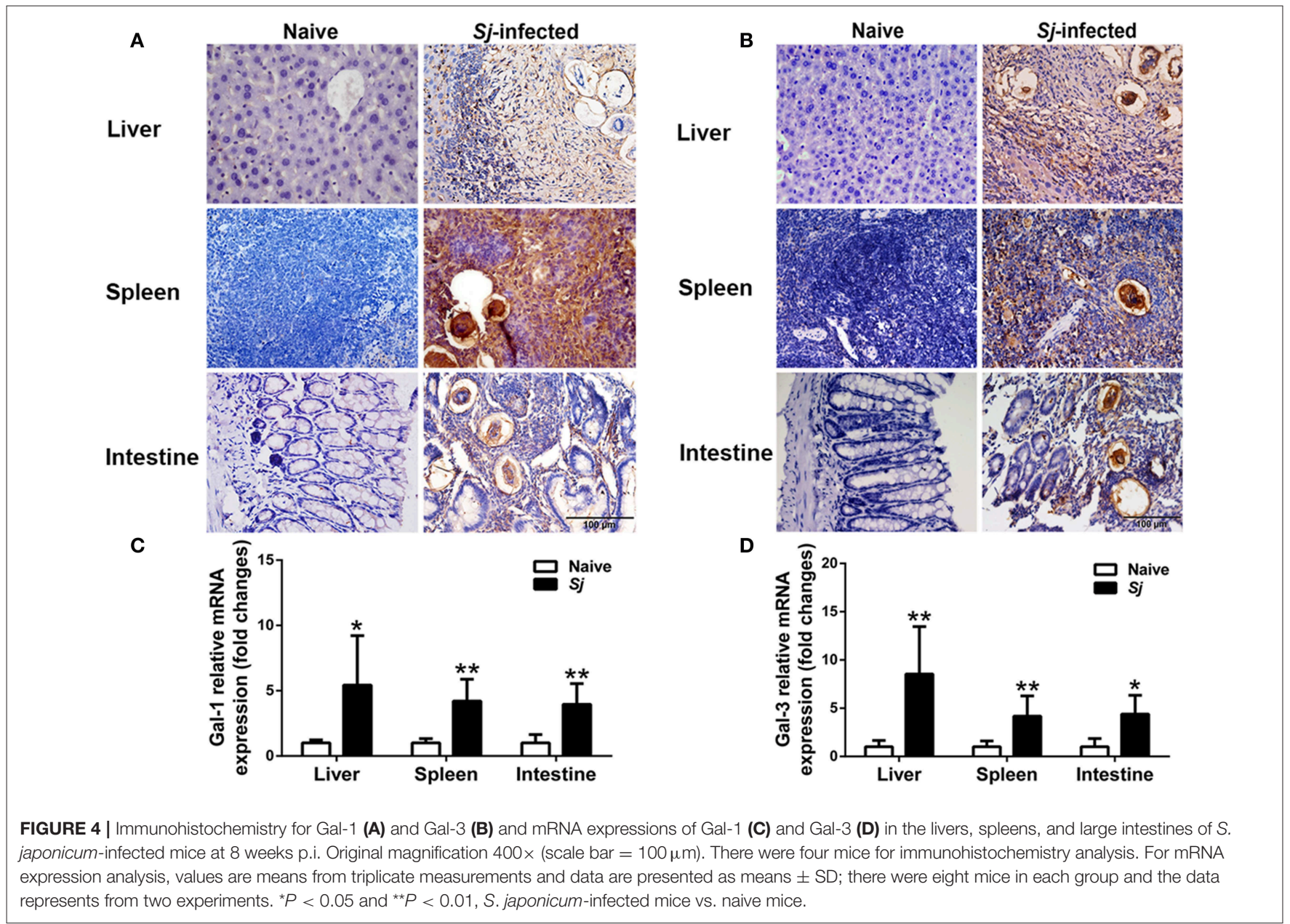


and large intestines $(P<0.01$ and $P<0.05$, respectively) of S. japonicum-infected mice at 8 weeks p.i. (Figures $4 \mathbf{C}, \mathbf{D}$ ).

\section{Immunohistochemical Staining for CD68 ${ }^{+}$ Macrophages and mRNA Expression} Levels of CD86, CD200R, IL-1 $\beta$, iNOS, Arg1, and Ym1 in the Livers, Spleens, and Large Intestines of S. japonicum-Infected Mice

Detected by immunohistochemical staining, compared with uninfected controls, there were more $\mathrm{CD} 68^{+}$macrophages around egg granulomas in the livers, spleens, and large intestines of S. japonicum-infected mice (Figure 5A). Quantitative analysis showed significantly higher CD68 macrophage-positive area in the livers, spleens, and large intestines of $S$. japonicuminfected mice $(P<0.001)$ in comparison with uninfected controls (Figure 5B). In addition, compared with uninfected controls, S. japonicum-infected mice presented significantly elevated mRNA levels of CD200R $(P<0.05)$, IL-1 $\beta(P<0.05)$, iNOS $(P<0.01)$, and Ym1 $(P<0.01)$ in the livers; elevated levels of Arg1 and Ym1 in the spleens $(P<0.01)$; and elevated levels of CD200R $(P<0.01), \operatorname{Arg} 1(P<0.05)$, and Ym1 $(P<0.05)$ in the large intestines at 8 weeks p.i. (Figure 5C).
The mRNA Expression Levels of CD86, CD200R, Gal-1, Gal-3, IL-1 $\beta$, iNOS, Arg1, and Ym1 in Peritoneal Macrophages of

\section{S. japonicum-Infected Mice}

Compared with uninfected controls, there were significantly elevated mRNA levels of CD200R $(P<0.01)$, Gal-1 $(P<0.001)$, Gal-3 $(P<0.001), \operatorname{Arg} 1(P<0.05)$, and $\mathrm{Ym} 1(P<0.01)$ in the peritoneal macrophages isolated from $S$. japonicum-infected mice at 8 weeks p.i. (Figure 6).

\section{The M2/M1 Ratios of CD200R/CD86, Ym1/IL-1 $\beta$, and Ym1/iNOS in the Livers, Spleens, Large Intestines, and Peritoneal Macrophages of S. japonicum-Infected Mice}

Compared with uninfected controls, S. japonicum-infected mice presented significantly elevated M2/M1 ratios of CD200R/CD86 $(P<0.001)$, Ym1/IL-1 $\beta(P<0.05)$, and $\mathrm{Ym} 1 / \mathrm{iNOS}(P<0.01)$ in the liver; elevated M2/M1 ratios of Ym1/IL-1 $\beta$ and $\mathrm{Ym} 1 / \mathrm{iNOS}$ in the spleen $(P<0.01)$; and elevated M2/M1 ratios of Ym1/IL$1 \beta(P<0.01)$ and $\mathrm{Ym} 1 / \mathrm{iNOS}(P<0.05)$ in the large intestine

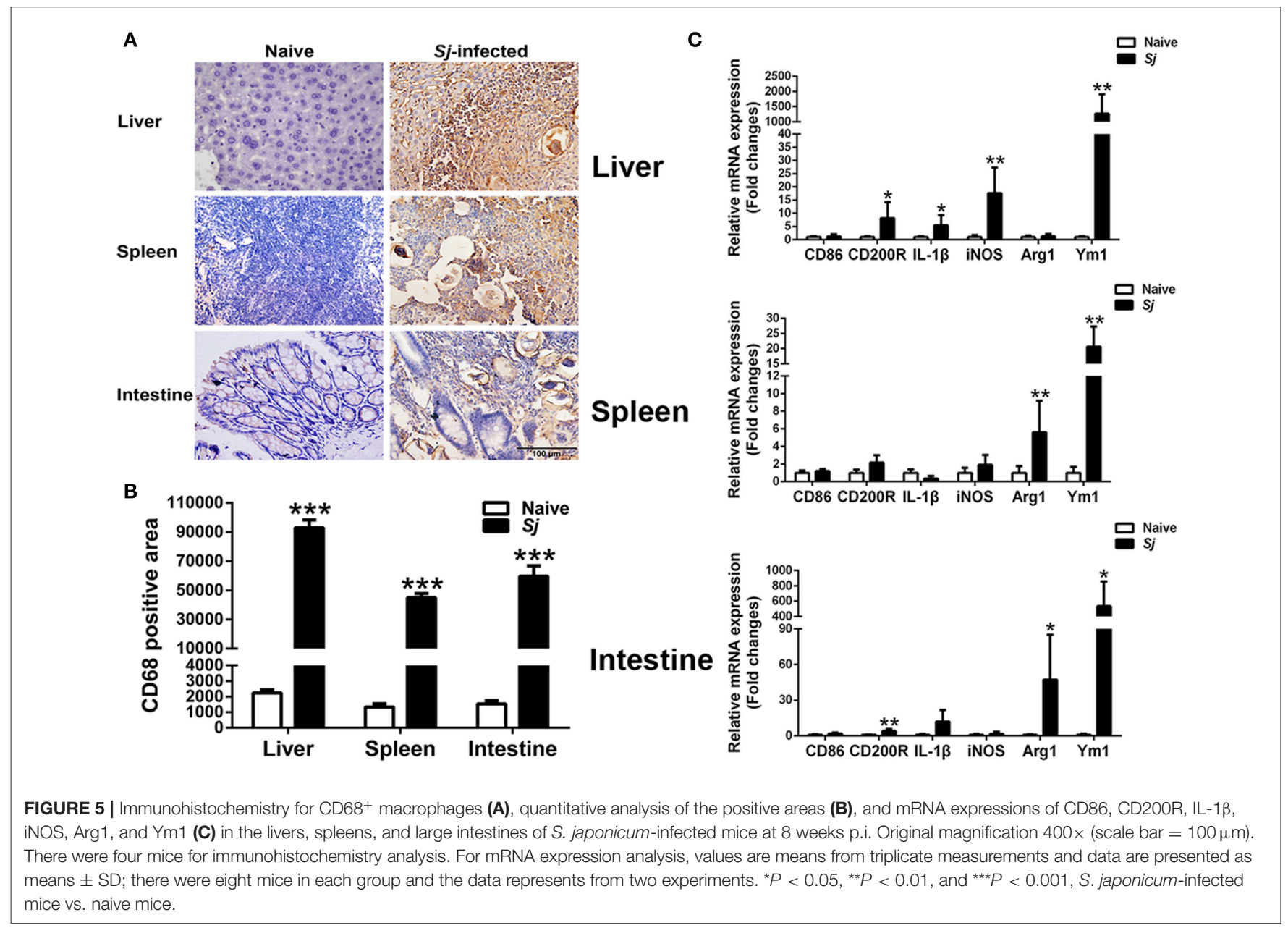




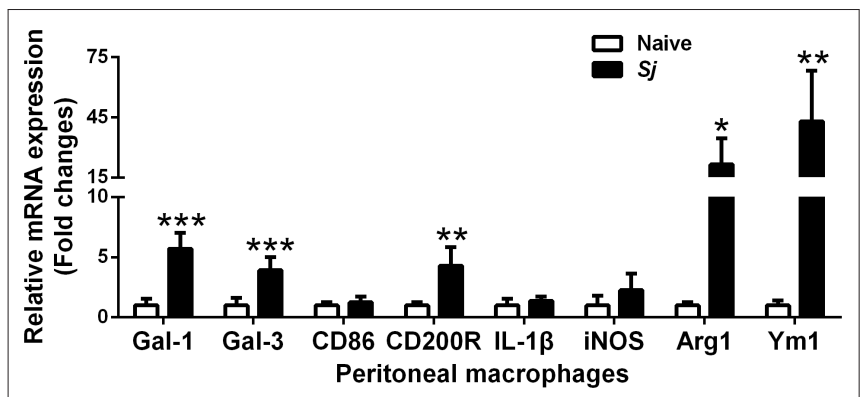

FIGURE 6 | The mRNA expressions of Gal-1, Gal-3, CD86, CD200R, IL-1 iNOS, Arg1, and Ym1 in peritoneal macrophages of S. japonicum-infected mice at 8 weeks p.i. Values are means from triplicate measurements and data are presented as means $\pm \mathrm{SD}$; there were eight mice in each group and the data represents from two experiments. ${ }^{\star} P<0.05$, ${ }^{\star \star} P<0.01$, and ${ }^{\star \star \star} P<$ 0.001, S. japonicum-infected mice vs. naive mice.

at 8 weeks p.i. Furthermore, there were significantly increased M2/M1 ratios of CD200R/CD86 $(P<0.05)$ and Ym1/IL-1 $\beta(P<$ $0.01)$ in the peritoneal macrophages isolated from $S$. japonicuminfected mice at 8 weeks p.i. (Figure 7).

\section{Correlation Analysis Between mRNA Expressions of Gal-1, Gal-3, EPX, and Ym1 in the Livers, Spleens, Large Intestines, and Peritoneal Macrophages of S. japonicum-Infected Mice}

The correlations between mRNA levels of different genes in the livers, spleens, large intestines, and peritoneal macrophages of $S$. japonicum-infected mice were analyzed. Only significant correlations were shown here. There were positive and significant correlations between Gal-1 and EPX $(r=0.961, P<0.001)$ and between Gal-3 and EPX $(r=0.881, P=0.004)$ in the livers, between Gal-3 and Ym1 in both the livers $(r=0.960$, $P<0.001)$ and large intestines $(r=0.966, P<0.001)$, and between Gal-3 and CD200R in the peritoneal macrophages $(r$ $=0.948, P<0.001)$ of $S$. japonicum-infected mice at 8 weeks p.i. (Figure 8).

\section{DISCUSSION}

Schistosomiasis is a chronic and potentially lethal disease, and the disease morbidity is caused by schistosome eggs and not directly by adult worms (35). Liver fibrosis caused by S. mansoni and $S$. japonicum is one of the most severe complications in advanced schistosomiasis, which usually accompanies portal hypertension and hepatosplenomegaly, and sometimes it is fatal (36). S. japonicum eggs can be deposited in ectopic locations, almost all organs and tissues of human host can be involved, and ectopic clinical manifestations and damage have been more frequently reported in patients with cerebrospinal and pulmonary schistosomiasis (37). So far, the understanding of host responses during $S$. japonicum infection remains inadequate. To address this scientific question, we used outbred Kunming mice to develop a mouse model of schistosomiasis japonica, which could thereby allow us to investigate the immune response of tissue pathology and consequent fibrosis caused by S. japonicum egg deposition.

Fibrosis is a common pathological outcome of most chronic inflammatory diseases, and both innate and adaptive immune responses contribute to the pathogenesis of fibrosis (38). Eosinophils participate in both innate and adaptive immune response to parasite through antibody-dependent cellular cytotoxicity and antigen-presenting function (5). The granules of eosinophils involve in the effect of antiparasite immunity, and ECP is important granule protein that acts as toxic to helminths $(39,40)$. During S. mansoni infection, an intense proliferation of eosinophils occurred in hepatic granuloma (41). Eosinophils have the role of destruction of S. mansoni eggs in vivo (42). S. haematobium egg-injected bladders of mice accumulate an eosinophil- and neutrophil-dominated mixed inflammatory infiltrate, and develop fibrosis and urinary dysfunction (43). Eosinophildeficient mice present markedly ameliorated radiation-induced small intestinal fibrosis, suggesting eosinophils as a crucial factor in the pathogenesis of radiation-induced intestinal fibrosis (44). In the present study, increased eosinophil infiltrations were observed in the granulomas in livers, spleens, and large intestines, which is the histologic hallmark of S. japonicum infection. Therefore, we addressed the question of whether egg granuloma can promote eosinophil degranulation. We focused on the expressions of CD69, CCL11, CCL24, ECP, and EPO in livers, spleens, and large intestines. Our data showed that after S. japonicum infection, a large number of eosinophils around granulomas in the livers, spleens, and large intestines of mice with advanced schistosomiasis japonica, as expected, the levels of CCL11 and CCL24 were increased in the liver, spleen, or large intestine. In addition, we found that the levels of CD69, ECP, and EPX were significantly increased in the livers, spleen, or large intestines, which indicated that eosinophil degranulation plays a crucial role in the development of egg granuloma formation and fibrosis induced by S. japonicum infection.

The pathogenesis of fibrosis is tightly regulated by macrophages in liver, lung, and gut (45). Macrophages produce inflammatory factors such as TGF- $\beta$ and plateletderived growth factor to activate HSCs and eventually lead to liver fibrosis, and macrophage polarization is associated with regulation of liver fibrosis (31). In an experiment, researchers combined a mouse model of urogenital schistosomiasis with macrophage depletion, and it has been demonstrated that macrophages modulate acute pathophysiology of S. haematobium egg-exposed bladder and necessary for the bladder fibrotic response to S. haematobium eggs (46). In the present study, our results showed that compared with uninfected mice, M1 macrophage markers (IL-1ß and iNOS) were only increased in liver; however, M2 macrophage markers (CD200R, Arg1, and Ym1) were increased in liver, spleen, large intestine, or peritoneal macrophages of mice with $S$. japonicum infection. In addition, the M2/M1 ratio of CD200R/CD86 in liver and peritoneal macrophages; the 

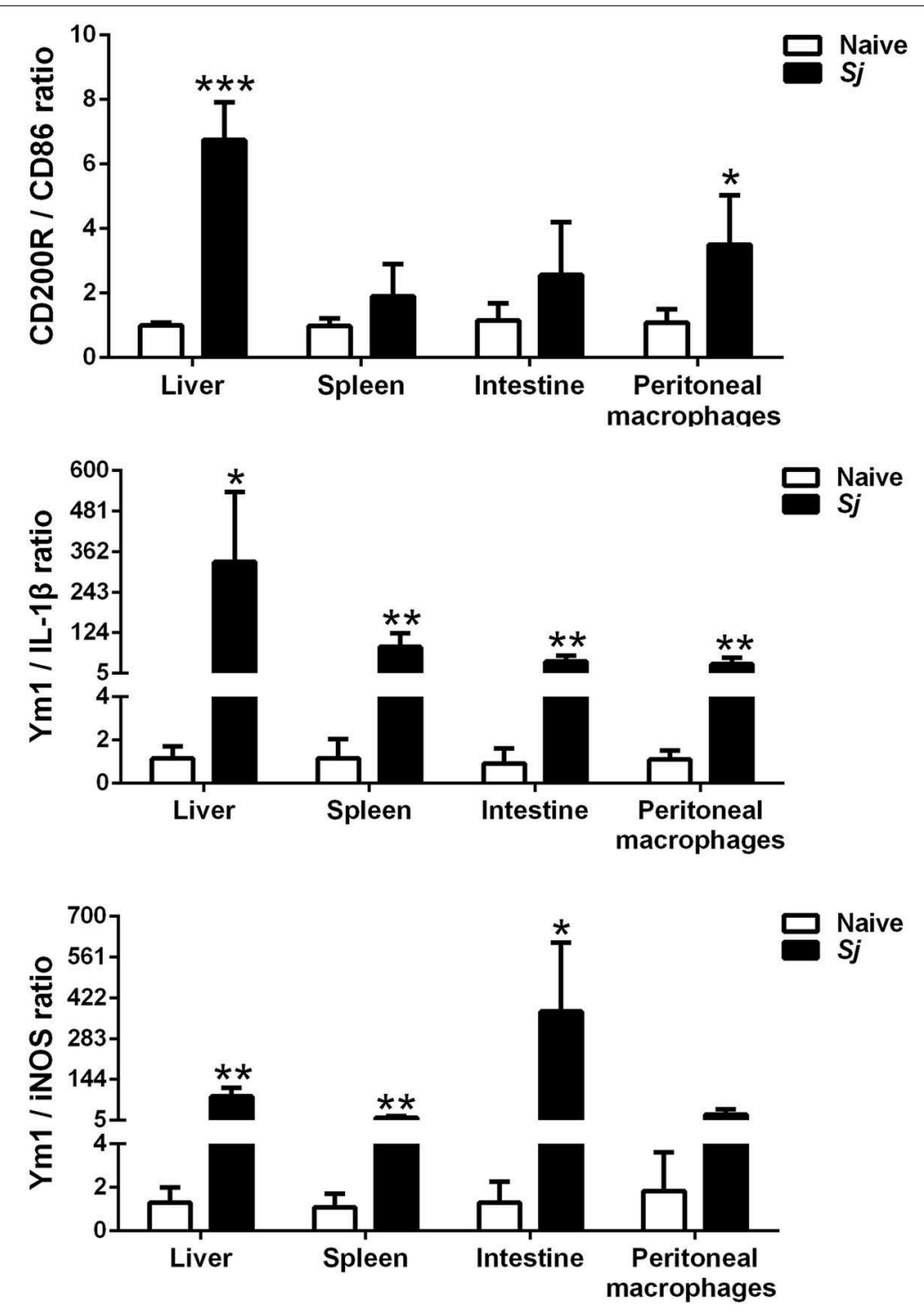

FIGURE 7 | The ratios of M2/M1 gene expression (CD200R/CD86, Ym1/IL-1 $\beta$, and Ym1/iNOS) in the livers, spleens, large intestines, and peritoneal macrophages of S. japonicum-infected mice at 8 weeks p.i. Data are presented as means $\pm \mathrm{SD}$; there were eight mice in each group and the data represents from two experiments. ${ }^{\star} P<0.05,{ }^{\star \star} P<0.01$, and ${ }^{\star \star \star} P<0.001$, S. japonicum-infected mice vs. naive mice.

ratios of Ym1/IL-1 $\beta$ and Ym1/iNOS in liver, spleen, and large intestine; and the ratio of Ym1/IL-1 $\beta$ in peritoneal macrophages were significantly increased in $S$. japonicum-infected mice at 8 weeks p.i. Schistosome worm antigen of S. japonicum facilitates the generation of M1 macrophages, whereas SEA preferentially promotes M2-polarized phenotype (47). It has been reported that peritoneal cavity is a secondary site of S. mansoni-associated inflammation, although it is not in direct contact with the parasite $(48,49)$. In mammals, tissue-resident macrophages are found all over the body in all organs and serous membranes, including the peritoneal cavity $(50,51)$. By detecting peritoneal macrophages from $S$. mansoni-infected mice, our data further demonstrated that M2 macrophage polarization plays a predominant role during murine chronic schistosomiasis japonica.
Galectins have been proven to modulate host responses against parasitic infection (52). Studies showed that Gal-1 knockdown can attenuate TGF- $\beta 1$-induced fibroblast activation in vitro (53) as well as reduce macrophages' polarization transform from M1 (pro-inflammatory) to M2 (antiinflammatory) during glioblastoma multiforme progression in a mouse model (54). Cell-surface glycans and Gal-1 promote HSC activation and migration; therefore, disrupting glycosylationdependent Gal-1/neuropilin-1 interactions may be a possible therapy for liver fibrosis (55). Gal-3 expression is up-regulated in human liver fibrosis and plays a critical role in liver fibrosis; animal study further demonstrated that myofibroblast activation is Gal-3 dependent (56). Use of Gal-3 inhibitor to cure liver fibrosis caused by non-alcoholic steatohepatitis has satisfied effect (34). Gal-3 directly binds SEA, and high levels of Gal-3 

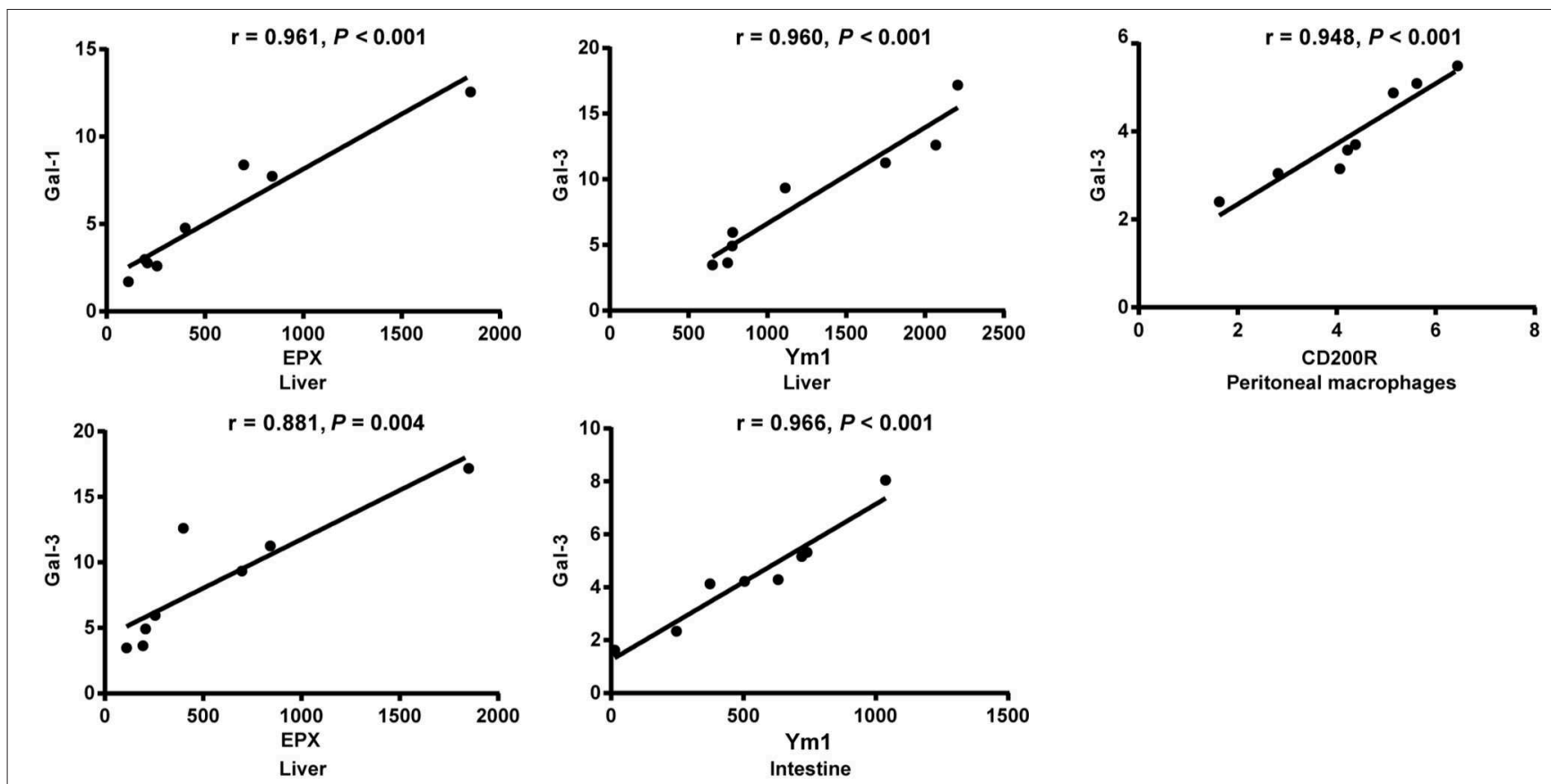

FIGURE 8 | Correlation analysis between the mRNA expressions of Gal-1/Gal-3 and EPX, Ym1, and CD200R in the livers, large intestines, or peritoneal macrophages of $S$. japonicum-infected mice at 8 weeks p.i. The $r$ value generates the theoretical line of best fit, and the $P$ value indicates the significance of the correlation. There were eight mice in each group and the data represents from two experiments.

can be found in the granulomas surrounding eggs of $S$. mansoni-infected hamsters (57). In the present study, our data demonstrated that both Gal-1 and Gal-3 were significantly increased in the livers, spleens, large intestines, and peritoneal macrophages of $S$. japonicum-infected mice at 8 weeks p.i., indicating that Gal-1 and Gal-3 play a role in the regulation of egg granuloma pathology during schistosomiasis japonica. Interestingly, we found significant correlations existed between Gal-1 and EPX and between Gal-3 and EPX in the liver, between Gal-3 and Ym1 in both the liver and large intestines, and between Gal-3 and CD200R in the peritoneal macrophages of mice suffered from schistosomiasis japonica. It has been found that Gal-3 is necessary for myofibroblast activation and fibrotic granulomas in $S$. mansoni-infected mice, and inflammatory infiltrate is amplified in Lgals $3^{-/-}$-infected mice in comparison with Lgals $3^{+/+}$-infected mice (33). The number of macrophages is decreased, and smaller granulomas and less collagen fibers are observed in the liver of Gal-3 $3^{-/}$mice infected with $S$. mansoni compared to infected wild-type mice, suggesting a direct correlation of Gal-3 with the fibrosis (32). Thus, Gal-1 and Gal-3 might be involved in egg granuloma fibrosis by regulating eosinophil degranulation and M2 macrophage polarization in advanced schistosomiasis japonica in the mouse model.

In conclusion, egg granuloma and fibrosis play very crucial roles in the pathological process induced by $S$. japonicum infection. Our study has proven that Gal-1, Gal-3, eosinophil and its granule proteins (ECP and EPX), and M2 macrophage may all play crucial roles in regulation of chronic immunopathology in murine schistosomiasis japonica. However, whether Gal-1 and Gal-3 contribute directly or indirectly to modulate S. japonicuminduced immune reaction and fibrosis through regulation of eosinophil degranulation and macrophages M2 polarization remains to be further determined.

\section{DATA AVAILABILITY STATEMENT}

The raw data supporting the conclusions of this article will be made available by the authors, without undue reservation, to any qualified researcher.

\section{ETHICS STATEMENT}

The animal study was reviewed and approved by the Animal Experimentation Ethics Committee of Zhongshan School of Medicine on Laboratory Animal Care at Sun Yat-sen University (No. 2016-081), and were carried out in strict accordance with institutional Guidelines for Care and Use of Laboratory Animals.

\section{AUTHOR CONTRIBUTIONS}

FL conceived and designed the experiments, analyzed the data, and wrote the manuscript. ZY performed the experiments and analyzed the data. SH edited the manuscript. YZ participated in analyzing the data. XM, HZ, ML, and JC participated in conducting the experiments. 


\section{FUNDING}

This work was supported by the Natural Science Foundation of China (no. 81971955); the Natural Science Foundation of Guangdong Province, China (no.

\section{REFERENCES}

1. Gordon CA, Kurscheid J, Williams GM, Clements ACA, Li Y, Zhou $\mathrm{XN}$, et al. Asian Schistosomiasis: Current Status and Prospects for Control Leading to Elimination. Trop Med Infect Dis. (2019) 4:40. doi: 10.3390/tropicalmed4010040

2. Gryseels B, Polman K, Clerinx J, Kestens L. Human schistosomiasis. Lancet. (2006) 368:1106-18. doi: 10.1016/S0140-6736(06)69440-3

3. Carson JP, Ramm GA, Robinson MW, McManus DP, Gobert GN. Schistosome-Induced Fibrotic Disease: The Role of Hepatic Stellate Cells. Trends Parasitol. (2018) 34:524-40. doi: 10.1016/j.pt.2018.02.005

4. Song LG, Wu XY, Sacko M, Wu ZD. History of schistosomiasis epidemiology, current status, and challenges in China: on the road to schistosomiasis elimination. Parasitol Res. (2016) 115:4071-81. doi: 10.1007/s00436-016-5253-5

5. Ravin KA, Loy M. The Eosinophil in Infection. Clin Rev Allergy Immunol. (2016) 50:214-27. doi: 10.1007/s12016-015-8525-4

6. Rumbley CA, Sugaya H, Zekavat SA, El Refaei M, Perrin PJ, Phillips SM. Activated eosinophils are the major source of Th2-associated cytokines in the schistosome granuloma. J Immunol. (1999) 162:1003-1009.

7. Olsson I, Venge P. Cationic proteins of human granulocytes. II. Separation of the cationic proteins of the granules of leukemic myeloid cells. Blood. (1974) 44:235-46. doi: 10.1182/blood.V44.2.235.235

8. Carlson MG, Peterson CG, Venge P. Human eosinophil peroxidase: purification and characterization. J Immunol. (1985) 134:1875-1879.

9. Peterson CG, Venge P. Purification and characterization of a new cationic protein-eosinophil protein-X (EPX)-from granules of human eosinophils. Immunology. (1983) 50:19-26.

10. Rasheed Z, Zedan K, Saif GB, Salama RH, Salem T, Ahmed AA, et al. Markers of atopic dermatitis, allergic rhinitis and bronchial asthma in pediatric patients: correlation with filaggrin, eosinophil major basic protein and immunoglobulin E. Clin Mol Allergy. (2018) 16:23. doi: 10.1186/s12948-018-0102-y

11. Matthews AN, Friend DS, Zimmermann N, Sarafi MN, Luster AD, Pearlman E, et al. Eotaxin is required for the baseline level of tissue eosinophils. Proc Natl Acad Sci U S A. (1998) 95:6273-8. doi: 10.1073/pnas.95.1 1.6273

12. Ganzalo JA, Jia GQ, Aguirre V, Friend D, Coyle AJ, Jenkins NA, et al. Mouse Eotaxin expression parallels eosinophil accumulation during lung allergic inflammation but it is not restricted to a Th2-type response. Immunity. (1996) 4:1-14. doi: 10.1016/S1074-7613(00)80293-9

13. Specht S, Saeftel M, Arndt M, Endl E, Dubben B, Lee NA, et al. Lack of eosinophil peroxidase or major basic protein impairs defense against murine filarial infection. Infect Immun. (2006) 74:5236-43. doi: 10.1128/IAI. 00329-06

14. Ramarokoto CE, Kildemoes AO, Randrianasolo BS, Ravoniarimbinina P, Ravaoalimalala VE, Leutscher $\mathrm{P}$, et al. Eosinophil granule proteins ECP and EPX as markers for a potential early-stage inflammatory lesion in female genital schistosomiasis (FGS). PLoS Negl Trop Dis. (2014) 8:e2974. doi: 10.1371/journal.pntd.0002974

15. Koller DY, Nilsson M, Enander I, Venge P, Eichler I. Serum eosinophil cationic protein, eosinophil protein $\mathrm{X}$ and eosinophil peroxidase in relation to pulmonary function in cystic fibrosis. Clin Exp Allergy. (1998) 28:241-8. doi: 10.1046/j.1365-2222.1998.00216.x

16. Paiva LA, Brand C, Bandeira-Melo C, Bozza PT, El-Cheikh MC, Silva PM, et al. Hepatic myofibroblasts derived from Schistosoma mansoni-infected mice are a source of IL-5 and eotaxin: controls of eosinophil populations in vitro. Parasit Vectors. (2015) 8:577. doi: 10.1186/s13071-015-1197-3
2019A1515011667); the Key Development Project of Graduate School of Sun Yat-sen University (no. cxjh201908), and the Undergraduate Teaching Reform Project of Zhongshan School of Medicine in 2019, Sun Yat-sen University, China.
17. Mangieri D, Corradi D, Martorana D, Malerba G, Palmisano A, Libri I, et al. Eotaxin/CCL11 in idiopathic retroperitoneal fibrosis. Nephrol Dial Transplant. (2012) 27:3875-84. doi: 10.1093/ndt/gfs408

18. Sica A, Mantovani A. Macrophage plasticity and polarization: in vivo veritas. J Clin Invest. (2012) 122:787-95. doi: 10.1172/JCI59643

19. Bility MT, Cheng L, Zhang Z, Luan Y, Li F, Chi L, et al. Hepatitis B virus infection and immunopathogenesis in a humanized mouse model: induction of human-specific liver fibrosis and M2-like macrophages. PLoS Pathog. (2014) 10:e1004032. doi: 10.1371/journal.ppat.1004032

20. Gordon S, Martinez FO. Alternative activation of macrophages: mechanism and functions. Immunity. (2010) 32:593-604. doi: 10.1016/j.immuni.2010.05.007

21. Xu J, Zhang H, Chen L, Zhang D, Ji M, Wu H, et al. Schistosoma japonicum infection induces macrophage polarization. J Biomed Res. (2014) 28:299-308.

22. Johannes L, Jacob R, Leffler H. Galectins at a glance. J Cell Sci. (2018) 131:jcs208884. doi: 10.1242/jcs.208884

23. Volarevic V, Markovic BS, Bojic S, Stojanovic M, Nilsson U, Leffler H, et al. Gal-3 regulates the capacity of dendritic cells to promote NKT-cell-induced liver injury. Eur J Immunol. (2015) 45:531-43. doi: 10.1002/eji.201444849

24. Hernandez JD, Baum LG. Ah, sweet mystery of death! Galectins and control of cell fate. Glycobiology. (2002) 12:127R-36R. doi: 10.1093/glycob/cwf081

25. Rabinovich GA, Toscano MA. Turning 'sweet' on immunity: galectin-glycan interactions in immune tolerance and inflammation. Nat Rev Immunol. (2009) 9:338-52. doi: 10.1038/nri2536

26. Perillo NL, Marcus ME, Baum LG. Galectins: versatile modulators of cell adhesion, cell proliferation, and cell death. J Mol Med (Berl). (1998) 76:402-12. doi: $10.1007 /$ s001090050232

27. Liu FT, Rabinovich GA. Galectins: regulators of acute and chronic inflammation. Ann N Y Acad Sci. (2010) 1183:158-82. doi: 10.1111/j.1749-6632.2009.05131.x

28. Noda Y, Kishino M, Sato S, Hirose K, Sakai M, Fukuda Y, et al. Galectin-1 expression is associated with tumour immunity and prognosis in gingival squamous cell carcinoma. J Clin Pathol. (2017) 70:126-33. doi: 10.1136/jclinpath-2016-203754

29. Maeda N, Kawada N, Seki S, Arakawa T, Ikeda K, Iwao H, et al. Stimulation of proliferation of rat hepatic stellate cells by galectin-1 and galectin- 3 through different intracellular signaling pathways. J Biol Chem. (2003) 278:18938-44. doi: 10.1074/jbc.M209673200

30. Jiang ZJ, Shen QH, Chen HY, Yang Z, Shuai MQ, Zheng SS. Galectin-1 gene silencing inhibits the activation and proliferation but induces the apoptosis of hepatic stellate cells from mice with liver fibrosis. Int J Mol Med. (2019) 43:103-16. doi: 10.3892/ijmm.2018.3950

31. Wynn TA, Vannella KM. Macrophages in Tissue Repair, Regeneration, and Fibrosis. Immunity. (2016) 44:450-62. doi: 10.1016/j.immuni.2016.02.015

32. Oliveira FL, Frazao P, Chammas R, Hsu DK, Liu FT, Borojevic R, et al. Kinetics of mobilization and differentiation of lymphohematopoietic cells during experimental murine schistosomiasis in galectin- $3^{-/-}$mice. J Leukoc Biol. (2007) 82:300-10. doi: 10.1189/jlb.1206747

33. de Oliveira FL, Carneiro K, Brito JM, Cabanel M, Pereira JX, Paiva LA, et al. Galectin-3, histone deacetylases, and Hedgehog signaling: Possible convergent targets in schistosomiasis-induced liver fibrosis. PLoS Negl Trop Dis. (2017) 11:e0005137. doi: 10.1371/journal.pntd.0005137

34. Traber PG, Zomer E. Therapy of experimental NASH and fibrosis with galectin inhibitors. PLoS One. (2013) 8:e83481. doi: 10.1371/journal.pone.0083481

35. Kamdem SD, Moyou-Somo R, Brombacher F, Nono JK. Host Regulators of Liver Fibrosis During Human Schistosomiasis. Front Immunol. (2018) 9:2781. doi: $10.3389 /$ fimmu.2018.02781 
36. Gray DJ, Ross AG, Li YS, McManus DP. Diagnosis and management of schistosomiasis. BMJ. (2011) 342:d2651. doi: 10.1136/bmj.d2651

37. Chen MG. Assessment of morbidity due to Schistosoma japonicum infection in China. Infect Dis Poverty. (2014) 3:6. doi: 10.1186/2049-9957-3-6

38. Wynn TA, Ramalingam TR. Mechanisms of fibrosis: therapeutic translation for fibrotic disease. Nat Med. (2012) 18:1028-40. doi: 10.1038/nm.2807

39. Bystrom J, Amin K, Bishop-Bailey D. Analysing the eosinophil cationic protein-a clue to the function of the eosinophil granulocyte. Respir Res. (2011) 12:10. doi: 10.1186/1465-9921-12-10

40. Hogan SP, Rosenberg HF, Moqbel R, Phipps S, Foster PS, Lacy P, et al. Eosinophils: biological properties and role in health and disease. Clin Exp Allergy. (2008) 38:709-50. doi: 10.1111/j.1365-2222.2008.02958.x

41. el-Cheikh MC, Dutra HS, Borojevic R. Eosinophil granulocyte proliferation and differentiation in schistosomal granulomas are controlled by two cytokines. Lab Invest. (1991) 64:93-7.

42. Olds GR, Mahmoud AA. Role of host granulomatous response in murine schistosomiasis mansoni. eosinophil-mediated destruction of eggs. J Clin Invest. (1980) 66:1191-9. doi: 10.1172/JCI109970

43. Fu CL, Odegaard JI, Herbert DR, Hsieh MH. A novel mouse model of Schistosoma haematobium egg-induced immunopathology. PLoS Pathog. (2012) 8:e1002605. doi: 10.1371/journal.ppat.1002605

44. Takemura N, Kurashima Y, Mori Y, Okada K, Ogino T, Osawa H, et al. Eosinophil depletion suppresses radiation-induced small intestinal fibrosis. Sci Transl Med. (2018) 10:eaan0333 doi: 10.1126/scitranslmed.aan0333

45. Wynn TA, Barron L. Macrophages: master regulators of inflammation and fibrosis. Semin Liver Dis. (2010) 30:245-57. doi: 10.1055/s-0030-1255354

46. Fu CL, Odegaard JI, Hsieh MH. Macrophages are required for host survival in experimental urogenital schistosomiasis. FASEB J. (2015) 29:193-207. doi: 10.1096/fj.14-259572

47. Zhu J, Xu Z, Chen X, Zhou S, Zhang W, Chi Y, et al. Parasitic antigens alter macrophage polarization during Schistosoma japonicum infection in mice. Parasit Vectors. (2014) 7:122. doi: 10.1186/1756-3305-7-122

48. Abdallahi OM, Bensalem H, Augier R, Diagana M, De Reggi M, Gharib B. Arginase expression in peritoneal macrophages and increase in circulating polyamine levels in mice infected with Schistosoma mansoni. Cell Mol Life Sci. (2001) 58:1350-7. doi: 10.1007/PL00000947

49. Tanabe M, Kaneko N, Takeuchi T. Schistosoma mansoni: suppression of carbamoyl phosphate synthetase (ammonia) and ornithine carbamoyltransferase activities in the liver of infected mice. Exp Parasitol. (1989) 68:432-42. doi: 10.1016/0014-4894(89)90128-8
50. Cohn ZA. Determinants of infection in the peritoneal cavity. I. Response to and fate of Staphylococcus aureus and Staphylococcus albus in the mouse. Yale J Biol Med. (1962) 35:12-28.

51. Ghosn EE, Cassado AA, Govoni GR, Fukuhara T, Yang Y, Monack DM, et al. Two physically, functionally, and developmentally distinct peritoneal macrophage subsets. Proc Natl Acad Sci USA. (2010) 107:2568-73. doi: $10.1073 /$ pnas.0915000107

52. Shi W, Xue C, Su XZ, Lu F. The roles of galectins in parasitic infections. Acta Trop. (2018) 177:97-104. doi: 10.1016/j.actatropica.2017.09.027

53. Lim MJ, Ahn J, Yi JY, Kim MH, Son AR, Lee SL, et al. Induction of galectin1 by TGF-betal accelerates fibrosis through enhancing nuclear retention of Smad2. Exp Cell Res. (2014) 326:125-35. doi: 10.1016/j.yexcr.2014.06.001

54. Van Woensel M, Mathivet T, Wauthoz N, Rosiere R, Garg AD, Agostinis $\mathrm{P}$, et al. Sensitization of glioblastoma tumor micro-environment to chemoand immunotherapy by Galectin-1 intranasal knock-down strategy. Sci Rep. (2017) 7:1217. doi: 10.1038/s41598-017-01279-1

55. Wu MH, Chen YL, Lee KH, Chang CC, Cheng TM, Wu SY, et al. Glycosylation-dependent galectin-1/neuropilin-1 interactions promote liver fibrosis through activation of TGF-beta- and PDGF-like signals in hepatic stellate cells. Sci Rep. (2017) 7:11006. doi: 10.1038/s41598-017-11212-1

56. Henderson NC, Mackinnon AC, Farnworth SL, Poirier F, Russo FP, Iredale JP, et al. Galectin-3 regulates myofibroblast activation and hepatic fibrosis. Proc Natl Acad Sci USA. (2006) 103:5060-5. doi: 10.1073/pnas.051 1167103

57. van den Berg TK, Honing H, Franke N, van Remoortere A, Schiphorst WE, Liu FT, et al. LacdiNAc-glycans constitute a parasite pattern for galectin-3-mediated immune recognition. J Immunol. (2004) 173:1902-7. doi: 10.4049/jimmunol.173.3.1902

Conflict of Interest: The authors declare that the research was conducted in the absence of any commercial or financial relationships that could be construed as a potential conflict of interest.

Copyright (c) 2020 Ye, Huang, Zhang, Mei, Zheng, Li, Chen and Lu. This is an open-access article distributed under the terms of the Creative Commons Attribution License (CC BY). The use, distribution or reproduction in other forums is permitted, provided the original author(s) and the copyright owner(s) are credited and that the original publication in this journal is cited, in accordance with accepted academic practice. No use, distribution or reproduction is permitted which does not comply with these terms. 